\title{
Pediatric Procedural Dermatology
}

\author{
Craig N. Burkhart, MD, MS, MPH
}

\section{PRACTICE POINTS}

- Children who require repetitive laser or surgical procedures over time benefit from regular monitoring of psychosocial needs.

- The informed consent process for children differs from adult procedural dermatology and should be adjusted to the maturity level of the patient.

- Common diagnoses unique to procedural pediatric dermatology that may require additional investigation include congenital melanocytic nevi, vascular anomalies, epidermal nevi, and midline lesions.

- Specific measures can be performed to improve outcomes when removing accessory tragi, dermoid cysts, pilomatricomas, and congenital nevi.

Pediatric procedural dermatology is a broad and emerging field. Pediatric patients often present with unique diagnoses, and procedures in this population often require special tools. In addition, performing procedures on infants, children, and teenagers requires special considerations, skill sets, and knowledge. This article provides a brief overview of decision-making processes, common diagnoses, and common procedures performed by dermatologists in this patient population.

Cutis. 2020;106:253-256.

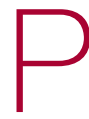

erforming dermatologic procedures in infants, children, and teenagers presents many unique challenges. There may be unique diagnoses, different instruments, differences in skin biology, or different approaches to pain management and anesthesia; the inclusion of a third party (caregivers) in decision processes; or a need to assess maturity level or to optimize outcomes over the patient's lifetime. The field of pediatric procedural dermatology is broad. This article reviews some of the more common procedures performed by pediatric dermatologists and some of the more common ethical and quality-of-life (QOL) considerations one might face in procedural pediatric dermatology. (The textbook Procedural Pediatric Dermatology ${ }^{1}$ offers a thorough discussion of this topic.)

\section{Quality of Life}

More often than not, procedures are performed in pediatric dermatology to improve QOL rather than to prevent morbidity or mortality. In the case of many self-limited conditions, such as ingrown nails or pyogenic granulomas, it is clear that intervention will improve the patient's QOL. In the case of warts and molluscum contagiosum, emotional, social, and cultural considerations play a large role in determining whether an intervention will improve QOL. Finally, some conditions, such as genodermatoses, giant congenital melanocytic nevi, and large vascular malformations, may be associated with additional systemic symptoms and may not have good treatment options for cure. In these cases, procedural interventions will result in a mixture of positive and negative QOL outcomes that can occur at the same time.

Bemmels et $\mathrm{al}^{2}$ published a qualitative study that provides a good foundation for understanding the positive and negative effects of procedural interventions on children and teenagers. In their study, children and teenagers who underwent reconstructive surgery for craniofacial differences noted improved self-esteem and reduced stigmatization. However, they also experienced negative outcomes, including an addiction to attaining a perfect surgical face, missing school for treatments, difficulty adjusting to an evolving appearance, anxiety related to not knowing when treatments will end, and experiencing stigma related to undergoing surgery. ${ }^{2}$ Thus, a comprehensive plan for the management of children who need ongoing procedures should include some level of

From the Department of Dermatology, The University of North Carolina at Chapel Hill, and Burkhart Pediatric \& Adolescent Dermatology, Cary, North Carolina.

The author reports no conflict of interest.

Correspondence: Craig N. Burkhart, MD, MS, MPH, 135 Parkway Office Ct, Ste 201, Cary, NC 27518 (pediatricdermatology@gmail.com). doi: $10.12788 /$ cutis.0104 
psychosocial support. Two good references on supporting young patients with visible differences include CBT for Appearance Anxiety: Psychosocial Interventions for Anxiety Due to Visible Difference ${ }^{3}$ and Reaching Teens: StrengthBased, Trauma-Sensitive, Resilience-Building Communication Strategies Rooted in Positive Youth Development. ${ }^{4}$

\section{Ethics}

Ethical decisions in pediatric procedural dermatology differ from adult dermatology in 3 major ways: (1) the involvement of a third party (ie, parents or legal guardians), (2) the need to assess the maturity of the patient, and (3) the need to know local laws in the jurisdiction in which care is being provided. Ethical dilemmas occur when the desires of the child, parents/guardians, and dermatologist are not in alignment. In these cases, it is important to be prepared with a moral or ethical framework to guide decision-making when conflicts occur. Two great resources are the best interest standard ${ }^{5}$ and the publication entitled, "Informed Consent in Decision-making in Pediatric Practice," from the American Academy of Pediatrics. ${ }^{6}$

In pediatrics, it often is better to conceptualize medical decision-making as a combination of informed permission and assent of the patient rather than informed consent. Informed permission describes how a parent or surrogate makes decisions for the child or adolescent and is similar to informed consent. A parent's informed permission may be in conflict with a child's wishes, but it is assumed that the parent is acting in the best interest of the child. Assent of the patient is the process of obtaining a minor's agreement to undergo an intervention even though he/she may lack legal authority or decisionmaking capacity to provide standard informed consent. It is important to respect the child's right to assent to interventions to the extent that their maturity level permits to develop trust with the dermatologist and medical encounters in general.

These differences emphasize an active process in which the patient, caregiver, and physician are all involved in the health care process and allow for increasing inclusion of the child as is developmentally appropriate. In the end, however, parents have the legal authority to give or withhold permission for a procedure. ${ }^{7}$ When this conflicts with a child's dissent, the dermatologist will need to objectively explore the reasons for the conflict and decide if a procedure is not in the child's best interests. If a mutual understanding cannot be reached between the dermatologist and parents, obtaining a second opinion is a good option. ${ }^{8}$

\section{Common Diagnoses}

The most common diagnoses unique to procedural pediatric dermatology include congenital melanocytic nevi, vascular anomalies, midline lesions, epidermal nevi, and pilomatricomas. Prior to intervening on these lesions, it is important to consider evaluating for associated diseases.
Congenital Melanocytic Nevi-Nevus Outreach has published best practices for the management of congenital melanocytic nevi. ${ }^{9}$ In newborns with a congenital melanocytic nevus greater than $3 \mathrm{~cm}$ in diameter or more than 20 satellite lesions, it is recommended that magnetic resonance imaging (MRI) of the brain and spine with and without gadolinium contrast be obtained before 6 months of age. Within the first 6 months of life, these children also should see ophthalmologists, neurologists, pediatric dermatologists, and plastic surgeons. These early referrals will help to establish a baseline for the patient and plan for possible interventions, if needed. Additionally, before 3 years of age, every child should be referred to psychology, even if he/she is asymptomatic. ${ }^{10}$

Vascular Anomalies-Prior to intervening on a vascular anomaly, it is important to accurately classify the lesion. Once the lesion is classified, an evaluation and treatment plan can be developed. The International Society for the Study of Vascular Anomalies has published a detailed classification guide that is a useful starting point in the management of vascular anomalies. ${ }^{11}$ Once a diagnosis is confirmed, further evaluation may include imaging, specialty referrals, genetic testing, biopsy, or blood tests, and a pediatric dermatologist usually helps to coordinate the care of patients with complex vascular anomalies.

Midline Lesions-Certain lesions in the midline may have a higher risk for neural tube dysraphism, and imaging should be performed prior to any procedural intervention. ${ }^{12}$ Midline cutaneous findings that are highly likely to be associated with dysraphism are lipomas, acrochordons, pseudotails, true tails, aplasia cutis congenita, congenital scars, dermoid cysts, dermoid sinuses, and infantile hemangiomas that are greater than $2.5 \mathrm{~cm}$ in diameter. An MRI should be performed for all high-risk lesions. Intermediate-risk lesions are atypical dimples ( $>5 \mathrm{~mm}$ in diameter or $>2.5 \mathrm{~cm}$ from the anal verge), hemangiomas less than $2.5 \mathrm{~cm}$ in diameter, and hypertrichosis. An ultrasound can screen for spinal dysraphism in these cases as long as imaging is performed prior to 6 months of age. If the child is older than 6 months, an MRI should be performed. Low-risk lesions that do not require imaging are simple dimples, hyperpigmentation, hypopigmentation, melanocytic nevi, port-wine stains, and telangiectases.

Epidermal Nevi-Children with epidermal nevi should have a complete physical examination, focusing on the skeletal system, central nervous system, and eyes. There are no specifically recommended imaging studies or referrals; however, several diagnostic clues can aid in the diagnosis of an epidermal nevus syndrome ${ }^{13}$ :

- Schimmelpenning syndrome: extensive nevus sebaceous and bowing or pain in the legs after 2 years of age

- Phacomatosis pigmentokeratotica: nevus sebaceous and nevus spilus

- Nevus comedonicus syndrome: ipsilateral cataract

- Angora hair nevus syndrome: soft white hair within the nevus 
- Becker nevus syndrome: breast hypoplasia

- Proteus syndrome: cerebriform plantar changes

- PIK3CA-related overgrowth spectrum: lipomas, macrodactyly, and/or vascular malformations

- Congenital hemidysplasia with ichthyosiform erythroderma and limb defects: inflammatory epidermal nevi, lateralization, ptychotropism, and ipsilateral limb defects

- Conradi-Hünermann-Happle syndrome: scaly red epidermal nevi without hair follicles and asymmetric limb shortening

Pilomatricomas-In addition to the tent signan angulated shape can be appreciated by stretching the skin overlying pilomatricomas-diagnosis of pilomatricoma can be confirmed by transillumination with an otoscope. In this case, a dark shadow typically is cast distal to where the otoscope touches the skin. ${ }^{14}$ In the case of multiple lesions, the patient should be evaluated for signs of myotonic dystrophy, Turner syndrome, and Gardner syndrome. ${ }^{15}$

\section{Common Procedures}

Pulsed Dye Laser-The pulsed dye laser is the most common laser used for red-colored lesions such as port-wine stains, facial telangiectases, and superficial hemangiomas. It also can be used to treat erythematous scars, verrucae, and psoriasis. In large vascular lesions, it typically is employed at 0.45 to 10 milliseconds every 4 to 6 weeks for 10 or more treatments. Port-wine stains preferably are treated within the first few months of life to provide the most fading without the need for general anesthesia. ${ }^{16}$ On the other hand, systemic therapy with propranolol is preferred over lasers for infantile hemangiomas. ${ }^{17}$

Long-Pulsed Alexandrite Laser (755 nm)-The alexandrite laser often is used to treat deeper vascular lesions such as venous lakes and hypertrophic port-wine stains. The operator needs to be cautious, as this laser has a higher incidence of scarring at the settings used to treat vascular lesions (typically fluences around $\left.60-85 \mathrm{~J} / \mathrm{cm}^{2}\right) .{ }^{18}$ It also may be used for hair reduction in disorders with hypertrichosis or hidradenitis suppurativa. ${ }^{19}$

Long-Pulsed Nd:YAG Laser-The long-pulsed Nd:YAG laser also can be used to treat deep vascular lesions and remove unwanted hair. Because of its low window of safety in the treatment of vascular lesions, the alexandrite laser usually is preferred. However, it is the preferred laser for treatment of unwanted hair and hidradenitis suppurativa in darker skin types. It often provides a $50 \%$ reduction in hair density after 9 treatments. ${ }^{20}$

Quality-Switched Lasers-Pigment granules in melanosomes and tattoo particles are targeted with quality-switched (QS) lasers. Typically, a device will contain a combination of QS 532-nm potassium-titanylphosphate (KTP) lasers, QS 1064-nm Nd:YAG lasers, and QS 755-nm alexandrite lasers in 1 machine. In general, shorter wavelengths are used to treat epidermal lesions such as ephelides, lentigines, and café-au-lait macules.
Longer wavelengths are used to treat deeper lesions such as nevus of Ota. A 2017 review suggested that café-au-lait macules with ragged borders (so-called coast of Maine borders) may respond well to QS lasers. ${ }^{21}$

Ablative Lasers-The 10,600-nm $\mathrm{CO}_{2}$ laser and 2940-nm erbium:YAG laser can be used to debulk superficial tumors such as lymphangiomas, syringomas, angiofibromas, and xanthomas. ${ }^{22}$ These treatments have a higher risk for scarring than other lasers, so it is important to have a good understanding of desired clinical end points before using these devices.

Fractionated Lasers-Fractionated lasers can be nonablative (several devices are available in the 1410- to 1927-nm range) or ablative $\left(\mathrm{CO}_{2}\right.$ or erbium:YAG). In pediatrics, they are usually used to treat burn scars, traumatic scars, and mild to moderate acne scarring. ${ }^{22}$ The most common side effects from fractionated lasers are prolonged erythema or hyperpigmentation. In addition, it typically takes at least 3 treatments to notice improvements.

\section{Excisions}

Pediatric procedural dermatologists remove a variety of unique lesions through excision. A few tips are provided for some of the more common lesions that may be excised in children.

Accessory Tragi-Prior to excising an accessory tragus, the surgeon should consider documenting a facial nerve examination, as accessory tragi can be associated with complete or partial facial nerve dysfunction. Additionally, there usually is an underlying cartilage structure present within the tragus. The cartilage stalk also should be addressed during the excision to avoid a continued palpable deformity after excision.

Dermoid Cysts-Dermoid cysts are the most commonly diagnosed benign orbital lesion in children. ${ }^{23}$ Exophytic periorbital lesions, which extend outside the orbital rim, can be removed through an infrabrow incision. Endophytic periorbital lesions, which are inside the orbital rim, should be removed through a crease incision. Midline lesions may have an intracranial extension and should be imaged through MRI and/or a computed tomography. ${ }^{24}$ Because dermoid cysts usually are located below the orbicularis oculi muscle, the muscle should be fixed with a suture prior to closing with skin sutures.

Pilomatricomas-Typically, a linear incision is made overlying the lesion, and then the underlying tumor is removed with sharp or blunt dissection. However, if the overlying skin has been stretched thin, a lenticular excision that includes the thinned skin may improve cosmesis.

Congenital Nevi-Large congenital nevi typically are removed through staged excisions. Lower extremity lesions are best removed before 10 months of age or before walking begins to minimize wound tension. However, if the procedure is not performed in infancy, it is best to wait until walking becomes stable. ${ }^{25}$ In older children, it is advisable to splint the affected lower extremity for 2 weeks to prevent dehiscence. The interval between 
excisions typically is 4 to 6 weeks for small lesions and 3 months for larger nevi.

\section{Conclusion}

Procedural pediatric dermatology is a broad and emerging field. As this article highlights, children are not small versions of adults and have unique biology, diseases, therapies, social situations, and ethical challenges from adults. This article provides a superficial overview of some of the more common issues faced by pediatric dermatologists and providers who perform procedures on infants, children, and teenagers. Readers who are interested in obtaining a more in-depth understanding of procedural pediatric dermatology should look at Procedural Pediatric Dermatology, ${ }^{1}$ the first textbook to provide expert opinion and evidence-based information on procedural management of pediatric skin conditions.

\section{REFERENCES}

1. Krakowski AC. Procedural Pediatric Dermatology. Phialdelphia, PA: Wolters Kluwer; 2011.

2. Bemmels H, Biesecker B, Schmidt J, et al. Psychological and social factors in undergoing reconstructive surgery among individuals with craniofacial conditions: an exploratory study. Cleft Palate Craniofac J. 2013;50:158-167.

3. Clarke A, Thompson AR, Jenkinson E, et al. CBT for Appearance Anxiety: Psychosocial Interventions for Anxiety Due to Visible Difference. Chichester, West Sussex: Wiley-Blackwell; 2013.

4. Ginsburg KR, Ramirez McClain ZB, eds. Reaching Teens: Strength-Based, Trauma-Sensitive, Resilience-Building Communication Strategies Rooted in Positive Youth Development. 2nd ed. Itasca, IL: American Academy of Pediatrics; 2020.

5. Kopelman LM. The best interests standard for incompetent or incapacitated patients of all ages. J Law Med Ethics. 2007;35:187-196.

6. Katz AL, Webb SA; Committee on Bioethics. Informed consent in decision-making in pediatric practice. Pediatrics. 2016;138:e20161485. doi:10.1542/peds.2016-1485.

7. Michon K. Emancipation of minors. NOLO website. https://www .nolo.com/legal-encyclopedia/emancipation-of-minors-32237.html. Accessed October 14, 2020

8. Cobb C, Bercovitch L. Ethical dilemmas. In: Krakowski AC, ed. Procedural Pediatric Dermatology. Philadelphia, PA: Wolters Kluwer; 2021:7-10.
9. Nevus Outreach, Inc., releases best practice guidelines [news release]. Bartlesville, OK: Nevus Outreach Inc; July 7, 2018. https://www.nevus.org/matrices/page_file_download.php?id=239. Accessed October 14, 2020.

10. Masnari O, Neuhaus K, Aegerter T, et al. Predictors of health-related quality of life and psychological adjustment in children and adolescents with congenital melanocytic nevi: analysis of parent reports. J Pediatr Psychol. 2019;44:714-725.

11. ISSVA classification for vascular anomalies. International Society for the Study of Vascular Anomalies website. https://www.issva.org /UserFiles/file/ISSVA-Classification-2018.pdf. Approved April 2014. Revised May 2018. Accessed October 14, 2020.

12. Sewell MJ, Chiu YE, Drolet BA. Neural tube dysraphism: review of cutaneous markers and imaging. Pediatr Dermatol. 2015;32:161-170.

13. Happle R. The group of epidermal nevus syndromes part I. well defined phenotypes. J Am Acad Dermatol. 2010;63:1-22.

14. Berreto-Chang OL, Gorell ES, Yamaguma MA, et al. Diagnosis of pilomatricoma using an otoscope. Pediatr Dermatol. 2010;27:554-557.

15. Danielson-Cohen A, Lin SJ, Hughes CA, et al. Head and neck pilomatrixoma in children. Arch Otolaryngol Head Neck Surg. 2001;127:1481-1483.

16. Jeon H, Bernstein LJ, Belkin DA, et al. Pulsed dye laser treatment of port-wine stains in infancy without the need for general anesthesia. JAMA Dermatol. 2019;155:435-441.

17. Krowchuk DP, Frieden IJ, Mancini AJ, et al. Clinical practice guideline for the management of infantile hemangiomas. Pediatrics. 2019;143:e20183475. doi:10.1542/peds.2018-3475.

18. Tierney EP, Hanke CW. Alexandrite laser for the treatment of port wine stains refractory to pulsed dye laser. Dermatol Surg. 2011;37:1268-1278

19. Alikhan A, Sayed C, Alavi A, et al. North American clinical management guidelines for hidradenitis suppurativa: a publication from the United States and Canadian Hidradenitis Suppurativa Foundations. J Am Acad Dermatol. 2019;81:76-90.

20. Rao K, Sankar TK. Long-pulsed Nd:YAG laser-assisted hair removal in Fitzpatrick skin types IV-VI. Lasers Med Sci. 2011;26:623-626.

21. Belkin DA, Neckman JP, Jeon H, et al. Response to laser treatment of café au lait macules based on morphologic features. JAMA Dermatol. 2017;153:1158-1161.

22. Kelly K, Lehmer L. Laser surgery. In: Krakowski AC, ed. Procedural Pediatric Dermatology. Philadelphia, PA: Wolters Kluwer; 2021:92-106.

23. Eldesouky MA, Elbakary MA. Orbital dermoid cyst: classification and its impact on surgical management. Semin Ophthalmol. 2018;33:170-174.

24. Pryor SG, Lewis JE, Weaver AL, et al. Pediatric dermoid cysts of the head and neck. Otolaryngol Head Neck Surg. 2005;132:938-942.

25. Metz BJ. Procedural pediatric dermatology. Dermatol Clin. 2013;31:337-346. 